\title{
Role of disturbed fatty acids metabolism in the pathophysiology of diabetic erectile dysfunction
}

\author{
Mohamed Raâfet Ben Khedher ${ }^{1 *}$, Houda Bouhajja ${ }^{2}$, Samia Haj Ahmed ${ }^{1}$, Mohamed Abid ${ }^{2}$, Kamel Jamoussi ${ }^{3}$ \\ and Mohamed Hammami ${ }^{1}$
}

\begin{abstract}
Background: Vasculogenic erectile dysfunction (VED) is considered as a common complication among people with type 2 diabetes (T2D). We tested whether changes in fatty acid (FAs) classes measured in erythrocytes are associated with increased risk of diabetic VED along with related risk factors.

Methods: We assessed erythrocyte FAs composition, lipid peroxidation parameters and inflammatory cytokines among 72 T2D men with VED, 78 T2D men without VED and 88 healthy volunteers with similar age. Biochemical, hepatic, lipid and hormonal profiles were measured.

Results: T2D people with VED had significant decrease in the indexes of $\triangle 6$-desaturase and elongase activities compared to the other studied groups. The same group of participants displayed lower erythrocytes levels of dihomo- $\gamma$ linolenic acid $(C 20: 3 n-6)(P<.001)$, precursor of the messenger molecule PGE1 mainly involved in promoting erection. Moreover, absolute SFAs concentration and HOMA IR levels were higher in T2D people with VED when compared to controls and associated with impaired NO concentration (1.43 vs $3.30 \mathrm{ng} / \mathrm{L}, P<.001)$. Our results showed that IL-6 and TNF-a were significantly increased and positively correlated with MDA levels only in T2D people with VED $(r=0.884, P=.016$ and $r=0.753, P=.035$; respectively) suggesting a decrease in the relative availability of vasodilator mediators and an activation of vasoconstrictors release.
\end{abstract}

Conclusion: Our findings show that the deranged FAs metabolism represents a potential marker of VED in progress, or at least an indicator of increased risk within men with T2D.

Keywords: Diabetic erectile dysfunction, Fatty acids, Inflammation, Insulin resistance, Type 2 diabetes

\section{Background}

Biomarkers of fatty acid (FAs) intake have been widely used in epidemiologic studies to predict risk of diseases [1]. The reliability and validity of diet records and food frequency questionnaire remain to be proved. Biomarkers of dietary FAs are objective, do not rely on the accuracy of memories and adequately reflect temporal changes in food composition, which could not be readily accommodated by classic methods of dietary assessment [1].

\footnotetext{
* Correspondence: med_benkhedher@hotmail.fr

${ }^{1}$ Laboratory of Nutrition, Functional Food and Vascular Health, Department of Biochemistry, Faculty of Medicine, University of Monastir, Avenue Avicenne, 5019 Monastir, Tunisia

Full list of author information is available at the end of the article
}

Type 2 diabetes (T2D) is determined chiefly by genes and lifestyle, yet dietary composition may affect both its development and complications [2]. FAs intake may increase diabetes complications risk since it influence glucose metabolism by altering cell enzyme activity, membrane function, gene expression, and insulin signaling [3]. Erectile dysfunction (ED) is one of the major complications of T2D [4]. The development of ED in men with diabetes is a multifactorial and complex process that appears to be affected by neurogenic and vascular causes, changes in nitric-oxide $(\mathrm{NO})$ system and oxidative processes [5].

Dysregulation of FAs metabolism is a key event responsible of insulin resistance (IR) and T2D [6]. In people with T2D, IR inhibits the activation and the expression of 
endothelial nitric oxide synthase (eNOS) and results in impaired NO bioactivity [7]. NO is released in vascular endothelium by activation of eNOS. Endothelialderived NO diffuses into adjacent vascular smooth muscle cells (VSMCs) where it reduces intracellular calcium $\left(\mathrm{Ca}^{2+}\right)$ bioavailability secondary to NO-mediated soluble guanylate cyclase (sGC) activation and cyclic guanosine 3 ',5' -monophosphate (cGMP) formation and leads to vasorelaxation by increased blood flow [8]. Erection can also be mediated by prostaglandin E1 (PGE1), a messenger molecule released by the cleavage of long chain $n-6$ dihomo- $\gamma$ linolenic acid (DGLA, C20:3) [9]. PGE1 activate the secretion of cyclic adenosine $3^{\prime}, 5^{\prime}$-monophosphate (cAMP) in VSMCs which regulates the activity of intracellular contractile proteins and promote the relaxation of penile VSMCs [9].

Oxidative stress plays a pivotal role in the development of diabetes complications. The involvement of lipid peroxidation in increased diabetes complications risk is clearly demonstrated; however no particular interest was given to its involvement in promoting ED. Free radical formation in diabetes by increased lipid peroxidation leads to damage of cellular machinery, enzymes and increased IR [10]. An excess of free radicals inactivates NO [11]. Yadav and Ramana (2013) showed that some lipid peroxidation products generated following oxidative processes modulate several pro-inflammatory cytokines gene expression [12]. Cytokines interact with VSMCs to activate potent vasoconstrictors release (protein kinase $\mathrm{C}$, rho-kinase) and to inhibit the synthesis of vasodilator mediators (NO, bradykinin) [13].

Recently, it was proposed that eicosapentaenoic acid (EPA, C20:5 n-3), docosahexaenoic acid (DHA, C22:6 $\mathrm{n}-3$ ) and their lipid mediator metabolites (resolvins and protectins) possess potent anti-inflammatory and protective effects in diverse diseases including diabetes and its related complications [14].

These different data suggest that substantial dysfunction of FAs metabolism in T2D may play an active role in the pathogenesis of ED. There are no published studies, to the best of our knowledge, which have examined the composition of FAs in red blood cells (RBCs) membranes and ED risk in diabetic patients. The present paper investigated whether changes in FAs metabolism are associated with VED susceptibility and related risk factors in people with T2D.

\section{Methods}

\section{Vasculogenic erectile dysfunction assessment}

The present work is a cross-sectional study of factors associated with a high risk for VED in men with T2D. All subjects diagnosed with T2D as per World Health Organization criteria completed a 5-item International Index of Erectile Function (IIEF-5) questionnaire [15].
IIEF-5 is a diagnostic tool used for the screening of erection problems and for assessing the severity of ED. Patients with a cutoff score of 21 underwent a penile color duplex Doppler ultrasonography test to establish the diagnosis of the type of vascular insufficiency. The assessment of penile vasculature was computed by measuring peak systolic velocity (PSV) in the deep penile arteries and end-diastolic velocity (EDV) after intracavernosal injection with $20 \mu \mathrm{g}$ prostaglandin E1. A PSV < $25 \mathrm{~cm} / \mathrm{s}$ or $25-30 \mathrm{~cm} / \mathrm{s}$ is considered to prove arterial insufficiency, a PSV $<25 \mathrm{~cm} / \mathrm{s}+\mathrm{EDV}>5 \mathrm{~cm} / \mathrm{s}$ indicate mixed vascular disorder and a PSV $>30 \mathrm{~cm} / \mathrm{s}+\mathrm{EDV} \leq$ $5 \mathrm{~cm} / \mathrm{s}$ represent non-vascular disorder [16].

\section{Study subjects}

Patients with any known thyroid dysfunction, hormonal disorder, malformations of the genitalia, Peyronie's disease, other sexual dysfunction including premature ejaculation or decreased libido, a history of pelvic surgery, kidney and liver failure, and any use of medication known to cause $E D$, such as antiandrogens, antihypertensive, antiarrhythmics, psychotropic drugs and antidepressants were excluded from the study [17]. According to the IIEF-5 score, the penile color ultrasound parameters and exclusion criteria, 72 men with T2D were diagnosed with VED.

A total of 78 T2D patients without VED were included in the study with respect to the excluding criteria. A number of 88 age-matched healthy volunteers without sexual dysfunction and who did not have diabetes or a history of, psychological, medical or neurological diseases, including ED, participated in this study.

\section{Ethics statement}

All participants gave consent to this study that was approved by the local Medical Ethics Committee of the Hedi Chaker Hospital of Sfax.

\section{Blood sampling and laboratory methods}

Blood samples were collected following overnight fasting. RBCs and plasma were separated by centrifugation. Biochemical, lipid and hepatic profiles were determined by automated analysis using the PLC ADVIA 1800 CHEMESTRY sYstem. Serum hormones were assessed using assay kits from Abbott laboratories (Chicago, USA) on the Architect Abbott ci4100 analyzer. HbA1c was measured by the PLC BioRAD D-10 (REF: 220-0101, D-10 ${ }^{\text {ma }}$ Hemoglobin A1C Reorder Pack). Cytokines (BMS213/2 and BMS223/4, eBioscience), insulin (REF:IS130D, Calbiotech) and NO (Cat.no.23479; Sigma) were quantified photometrically using immunoenzymetric assay kits. MDA and conjugated dienes were determined as described previously $[18,19]$. 
Fatty acids extraction and gas chromatography (GC) analysis

Lipid extraction for quantitative analysis of FAs classes was performed as reported previously [20]. Total lipids fractions were converted into methyl esters using 14\% methanolboron trifluoride. The FAs methyl esters were separated, identified and determined by gas chromatography (GC) using a Hewlett Packard 5890 II GC equipped with Flame Ionization Detector and HPINNOWax capillary column (5\% phenyl/95\% dimethylpolysiloxane: $30 \mathrm{~m} \times 0.25 \mathrm{~mm}$, i.d., film thickness $0.25 \mu \mathrm{m})$. The concentration of each individual FAs was expressed as a relative percentage of total area under the peaks according to Zarrouk et al. [21].

\section{Statistical analysis}

The Statistical Package for the Social Sciences (SPPS 18.0 for Windows) was used to perform all statistical analysis. Student's unpaired $t$-test was used to analyze clinical characteristics. Pearson's chi-squared test with Fisher's exact probability was used to compare categorical variables of subject's baseline data. MannWhitney U-test was used in cases that did not have a normal distribution. The difference was considered significant at $P<.05$.

\section{Results}

Baseline and specific cohort characteristics

The main clinical features, biochemical, inflammatory and hormonal parameters of the studied groups are summarized (Tables 1 and 2). As a known risk factor for $\mathrm{ED}$, age was matched to patients and healthy volunteers groups. A statistically significant increase was observed in both T2D patient groups (Group A: T2D with VED and group B: T2D without VED) compared to the controls (group C) on the rate of fasting glucose, HbAlc, insulin, HOMA IR, total cholesterol and LDL-cholesterol plasmatic levels. However, a significant decrease in both patient groups was observed on ALT levels, when compared to controls. AST concentration was statistically reduced only in patients of group B whereas triglycerides levels were higher in the same group of patients compared to the control group.

Mean concentrations of MDA, IL- 6 and TNF- $\alpha$ were significantly higher in group A than the control group (Table 2). Our results showed that IL-6 and TNF- $\alpha$ were positively correlated with MDA levels only in T2D patients of group A $(r=0.884, P=.016$ and $r=0.753, P$ $=.035$; respectively) (Table 3 ). The group $\mathrm{A}$ also displayed lower plasmatic levels of NO compared to the other groups. No differences in conjugated diene

Table 1 Clinical and biochemical characteristics of the study participants

\begin{tabular}{|c|c|c|c|}
\hline Characteristics & $\begin{array}{l}\text { Patients with VED } \\
(n=72)\end{array}$ & $\begin{array}{l}\text { Patients w/o VED } \\
(n=78)\end{array}$ & $\begin{array}{l}\text { Controls } \\
(n=88) \\
\end{array}$ \\
\hline \multicolumn{4}{|l|}{ Clinical profile } \\
\hline Age (years) & $57.7 \pm 5.1$ & $58.8 \pm 4.8$ & $55.9 \pm 7.0$ \\
\hline BMI $\left(\mathrm{kg} / \mathrm{m}^{2}\right)$ & $26.75 \pm 4.8$ & $27.43 \pm 3.2$ & $25.15 \pm 3.8$ \\
\hline Duration of T2D (years) & $10.95 \pm 7.1$ & $8.25 \pm 5.4$ & - \\
\hline Duration of ED (years) & $3.30 \pm 2.9$ & - & - \\
\hline \multicolumn{4}{|l|}{ Biochemical profile } \\
\hline Fasting Glucose (mmoL/L) & $9.87 \pm 3.7^{* *}$ & $8.36 \pm 2.1^{* *}$ & $5.27 \pm 0.9$ \\
\hline HbA1c (mmol/mol) & $95^{* *}$ & $77^{* *}$ & 38 \\
\hline Insulin ( $\mu \mid \mathrm{U} / \mathrm{mL})$ & $5.62 \pm 3.6^{*}$ & $4.93 \pm 2.7^{*}$ & $3.56 \pm 2.4$ \\
\hline HOMA IR & $2.39 \pm 1.7^{* *}$ & $1.83 \pm 1.1^{* *}$ & $0.83 \pm 0.4$ \\
\hline $\mathrm{NO}(\mathrm{ng} / \mathrm{L})$ & $1.43 \pm 1.18^{* * *}$ & $3.09 \pm 1.85$ & $3.30 \pm 2.92$ \\
\hline \multicolumn{4}{|l|}{ Serum lipid profile (mmoL/L) } \\
\hline Total cholesterol & $4.54 \pm 0.8^{*}$ & $4.43 \pm 0.8^{*}$ & $4.06 \pm 0.9$ \\
\hline Triglycerides & $1.60 \pm 0.9$ & $1.77 \pm 0.7^{*}$ & $1.37 \pm 0.5$ \\
\hline LDLC & $2.70 \pm 0.94^{*}$ & $2.68 \pm 0.9^{*}$ & $2.27 \pm 1,02$ \\
\hline HDLC & $1.03 \pm 0.3$ & $0.94 \pm 0.4$ & $1.1 \pm 0.4$ \\
\hline \multicolumn{4}{|l|}{ Serum Hepatic profile (IU/L) } \\
\hline AST & $23.19 \pm 7.5$ & $21.5 \pm 8.4^{*}$ & $24.28 \pm 8.9$ \\
\hline ALT & $20.26 \pm 11.6^{*}$ & $21.36 \pm 10.4^{*}$ & $25.01 \pm 12.3$ \\
\hline
\end{tabular}

Values are given as mean \pm standard error

$B M I$ Body mass index, HbA1c glycated hemoglobin, HOMA IR homeostatic index of insulin resistance, $L D L C$ low density lipoprotein-cholesterol, HDLC High density lipoprotein-cholesterol, $A S T$ aspartate aminotransferase, $A L T$ alanine aminotransférase, w/o without

${ }^{*} P<0.05$ and ${ }^{* *} P<0.001$ compared to controls 
Table 2 Specific clinical characteristics of studied subjects

\begin{tabular}{|c|c|c|c|}
\hline Characteristics & $\begin{array}{l}\text { Patients with VED } \\
(n=72)\end{array}$ & $\begin{array}{l}\text { Patients w/o ED } \\
(n=78)\end{array}$ & $\begin{array}{l}\text { Controls } \\
(n=88)\end{array}$ \\
\hline \multicolumn{4}{|l|}{ Inflammatory Cytokines } \\
\hline IL-6 (pg/mL) & $0.86 \pm 0.5^{* *}$ & $0.51 \pm 0.2$ & $0.44 \pm 0.3$ \\
\hline TNF-a $(p g / m L)$ & $3.3 \pm 2.9^{* *}$ & $2.9 \pm 1.9^{* *}$ & $1.43 \pm 1.2$ \\
\hline \multicolumn{4}{|l|}{ Lipid peroxidation } \\
\hline $\mathrm{MDA}(\mu \mathrm{M})$ & $23.9 \pm 4.5^{* *}$ & $18.7 \pm 5.3$ & $17.11 \pm 5.9$ \\
\hline Conjugated diene $(\mu \mathrm{M})$ & $139.16 \pm 68.4$ & $141.76 \pm 34.5$ & $122.4 \pm 55.9$ \\
\hline \multicolumn{4}{|l|}{ Serum Hormones } \\
\hline $\mathrm{FSH}(\mathrm{mlU} / \mathrm{mL})$ & $7.22 \pm 4.9$ & $6.83 \pm 2.6$ & $6.32 \pm 2.4$ \\
\hline $\mathrm{LH}(\mathrm{mlU} / \mathrm{mL})$ & $5.67 \pm 3.4$ & $6.14 \pm 3.6$ & $6.07 \pm 2.1$ \\
\hline PRL (ng/mL) & $12.16 \pm 5.1$ & $12.83 \pm 4.4$ & $11.19 \pm 2.9$ \\
\hline Testosterone (ng/mL) & $5.35 \pm 1.5$ & $6.12 \pm 2.8$ & $5.89 \pm 2.1$ \\
\hline FT4 (pmoL/L) & $13.92 \pm 1.5$ & $12.53 \pm 2.6$ & $13.64 \pm 2.3$ \\
\hline hsTSH ( $\mu \mathrm{IU} / \mathrm{mL})$ & $1.95 \pm 0.9$ & $1.76 \pm 0.7$ & $1.83 \pm 0.9$ \\
\hline
\end{tabular}

Values are given as mean \pm standard error

NO nitric oxide, TNF- $a$ tumor necrosis factor alpha, IL-6 interleukine 6, MDA malondialdehyde, FSH follicle-stimulating hormone, $L H$ luteinizing hormone, $P R L$ prolactin, FT4 Free thyroxine 4, hsTSH high sensitive thyroid-stimulating hormone, w/o without

${ }^{*} P<0.05$ and ${ }^{* *} P<0.001$ compared to controls

concentrations and all tested serum hormones (FSH, LH, PRL, testosterone, FT4 and hsTSH) were recorded between the three studied groups.

\section{Evaluation of fatty acid profile in red blood cells}

The FAs profile of RBC membranes and the indexes of the microsomal enzymes systems activities are given in Table 4. Analysis of FAs composition in people with $\mathrm{T} 2 \mathrm{D}$ of group A and B showed a significant increase in the levels of very long chain fatty acids (VLCFA) such as behenic acid (C22:0), lignoceric acid (C24:0) and cerotic acid (C26:0) compared to healthy subjects. Besides, T2D patients of group A presented higher total saturated fatty acids (SFAs) content than patients of group $B$ and the controls mainly due to the increase of VLCFA, myristic and palmitic acids. Meanwhile, total monounsaturated FAs (MUFAs) concentration was lower in T2D patients

Table 3 Univariate correlation of malondialdehyde with inflammatory cytokines levels among the study population

\begin{tabular}{llll}
\hline $\begin{array}{lll}\text { Inflammatory } \\
\text { Cytokines }\end{array}$ & Studied Subjects & \multicolumn{2}{l}{ Correlation with MDA } \\
\cline { 3 - 4 } IL-6 & Patients with VED & 0.884 & $0.016^{*}$ \\
& Patients w/o VED & -0.184 & 0.497 \\
& Controls & -0.121 & 0.312 \\
TNF-a & Patients with VED & 0.753 & $0.035^{*}$ \\
& Patients w/o VED & 0.497 & 0.078 \\
& Controls & -0.116 & 0.333 \\
\hline
\end{tabular}

IL-6 interleukine 6, TNF- $a$ tumor necrosis factor alpha, MDA malondialdehyde, $r$ correlation coefficients, $w / o$ without ${ }^{*} P<0.05$ of group A and B than in controls with most particularly a decrease in myristoleic acid (C14:1 n-5), oleic acid (C18:1 n-9) and eicosenoic acid (C20:1 n-9).

The data on the composition of FAs subtypes of PUFAs showed significant changes, however absolute PUFAs concentration was similar in all groups. The differences reported in this lipid fraction were primarily due to the increases of $\omega 3 \alpha$-linolenic acid (ALA, C18:3 $n-3)$ and of $\omega 6$ linoleic acid (LA, C18:2 n-6) in patients of group A compared to controls. However, a significant decrease was recorded in the same group of patients regarding erythrocytes concentration of dihomo- $\gamma$ linolenic acid (DGLA, C20:3 n-6), EPA (C20:5 n-3) and DHA (C22:6 n-3).

The desaturation indexes of enzymes activities were assessed as described previously [20]. The elongase activity was statistically lower in patients of group A and B compared to the control group. The index of $\Delta 6$ desaturase activity was substantially reduced only in patients with VED compared to the control subjects. A significant increase was noted for $\Delta 5$-desaturase activity in both T2D patients groups in comparison to healthy volunteers. No variations were recorded for the $\Delta 9$ desaturase activity between the studied groups.

\section{Additional study variables}

The patients were divided into four groups according to the severity of ED: 7 (9.8\%) had light ED, 29 (40.4\%) had mild ED, 24 (33.4\%) had moderate ED and 12 (16.4\%) had severe ED. The distribution of FAs classes according to ED severity was assessed using a multivariate analysis. No significant associations were recorded between the 
Table 4 Erythrocytes fatty acid profiles of the study population determined by gas chromatography

\begin{tabular}{|c|c|c|c|c|}
\hline $\begin{array}{l}\text { Fatty Acids } \\
\text { (nomenclature) }\end{array}$ & Name & $\begin{array}{l}\text { Patients with VED (\%) } \\
(n=72)\end{array}$ & $\begin{array}{l}\text { Patients w/o VED (\%) } \\
(n=78)\end{array}$ & $\begin{array}{l}\text { Controls (\%) } \\
(n=88)\end{array}$ \\
\hline C12: 0 & Lauric acid & $0.21 \pm 0.12$ & $0.19 \pm 0.11$ & $0.21 \pm 0.13$ \\
\hline C13: 0 & Tridecylic acid & $0.79 \pm 0.53^{* *}$ & $0.47 \pm 0.45$ & $0.53 \pm 0.35$ \\
\hline C14: 0 & Myristic acid & $4.12 \pm 2.50^{* * *}$ & $3.03 \pm 2.09^{*}$ & $2.57 \pm 2.30$ \\
\hline C15: 0 & Pentadecylic acid & $1.07 \pm 0.69^{* * *}$ & $0.75 \pm 0.45^{*}$ & $0.63 \pm 0.51$ \\
\hline C16: 0 & Palmitic acid & $28.82 \pm 5.75^{* *}$ & $29.25 \pm 6.66^{* *}$ & $25.87 \pm 4.16$ \\
\hline C18: 0 & Stearic acid & $11.93 \pm 5.82^{* * *}$ & $13.29 \pm 5.18^{*}$ & $14.88 \pm 5.15$ \\
\hline C20: 0 & Arachidic acid & $0.24 \pm 0.32$ & $0.23 \pm 0.13^{*}$ & $0.26 \pm 0.31$ \\
\hline C22: 0 & Behenic acid & $0.43 \pm 0.41^{* * *}$ & $0.35 \pm 0.16^{* *}$ & $0.27 \pm 0.21$ \\
\hline C23: 0 & Tricosylic acid & $0.59 \pm 0.42$ & $0.44 \pm 0.28$ & $0.60 \pm 0.79$ \\
\hline C24: 0 & Lignoceric acid & $2.06 \pm 0.83^{* *}$ & $1.75 \pm 1.14$ & $1.42 \pm 1.19$ \\
\hline C26: 0 & Cerotic acid & $5.47 \pm 2.28^{* * *}$ & $3.21 \pm 3.05^{* *}$ & $3.72 \pm 2.81$ \\
\hline$\Sigma$ SFA & & $55.70 \pm 9.4^{* *}$ & $52.84 \pm 7.1$ & $51,01 \pm 8.7$ \\
\hline C14:1 cis-9 (n-5) & Myristoleic acid & $1.33 \pm 0.71^{* *}$ & $0.98 \pm 0.44^{* * *}$ & $1.60 \pm 0.56$ \\
\hline C16:1 cis-9 (n-7) & Palmitoleic acid & $0.56 \pm 0.51$ & $0.60 \pm 0.54$ & $0.57 \pm 0.47$ \\
\hline C18:1 cis-9 (n-9) & Oleic acid & $10.96 \pm 4.18^{* * *}$ & $13.14 \pm 3.31^{*}$ & $14.53 \pm 3.65$ \\
\hline C18:1 cis $11(n-7)$ & Vaccenic acid & $1.31 \pm 0.30$ & $1.39 \pm 0.38$ & $1.47 \pm 0.49$ \\
\hline C20:1 cis11 (n-9) & Eicosenoic acid & $0.16 \pm 0.13^{* * *}$ & $0.22 \pm 0.18^{*}$ & $0.26 \pm 0.17$ \\
\hline C22:1 cis13(n-9) & Erucic acid & $0.45 \pm 0.21$ & $0.50 \pm 0.21$ & $0.54 \pm 0.87$ \\
\hline C24:1 (n-9) & Nervonic acid & $1.70 \pm 0.59^{* * *}$ & $1.61 \pm 0.97^{* * *}$ & $3.00 \pm 1.31$ \\
\hline$\Sigma$ MUFA & & $16.50 \pm 4.12^{* * *}$ & $18.43 \pm 3.65^{* * *}$ & $21.00 \pm 3.88$ \\
\hline \multicolumn{5}{|l|}{ Omega 3 family (n-3) } \\
\hline C18:3 cis9,12,15 & a-linolenic acid & $0.66 \pm 0.34^{* * *}$ & $0.63 \pm 0.27^{* * *}$ & $0.51 \pm 0.28$ \\
\hline C20:3 cis $11,14,17$ & Eicosatrienoic acid & $1.43 \pm 0.48$ & $1.80 \pm 0.83^{*}$ & $1.61 \pm 0.95$ \\
\hline C20:5 EPA & Eicosapentaenoic acid & $0.52 \pm 0.26^{* * *}$ & $1.26 \pm 1.02$ & $1.54 \pm 1.92$ \\
\hline C22:6 DHA & Docosahexaenoic acid & $2.00 \pm 1.39^{* * *}$ & $2.27 \pm 1,21^{* * *}$ & $3.13 \pm 1.20$ \\
\hline \multicolumn{5}{|l|}{ Omega 6 family (n-6) } \\
\hline C18:2 cis9,12 & Linoleic acid & $12.05 \pm 4.46^{* * *}$ & $10.40 \pm 3.20^{* *}$ & $9.40 \pm 5.21$ \\
\hline C18:3 cis6,9,12 & y-linolenic acid & $0.16 \pm 0.17^{*}$ & $0.19 \pm 0.14$ & $0.17 \pm 0.11$ \\
\hline C20:3 cis $8,11,14$ & Dihomo-plinolenic acid & $0.18 \pm 0.09^{* * *}$ & $0.28 \pm 0.16$ & $0.38 \pm 0.27$ \\
\hline C20:4 & Arachidonic acid & $10.75 \pm 3.09^{*}$ & $11.43 \pm 2.38^{* * *}$ & $9.25 \pm 3.36$ \\
\hline$\Sigma$ PUFA & & $27.79 \pm 5.06$ & $28.42 \pm 4.11$ & $26.03 \pm 5.85$ \\
\hline$\Sigma$ UFA & & $44.30 \pm 5.75^{* *}$ & $47.16 \pm 6.03$ & $47.03 \pm 6.75$ \\
\hline \multicolumn{5}{|l|}{ Desaturation index } \\
\hline C20:4/C20:3 ( $\triangle 5 \mathrm{D})$ & & $77.03 \pm 51.26^{* * *}$ & $54.18 \pm 40.75^{*}$ & $47.99 \pm 43.92$ \\
\hline C18:3/C18:2n-6 ( $\triangle 6 \mathrm{D})$ & & $0.015 \pm 0.016^{* *}$ & $0.020 \pm 0.015$ & $0.023 \pm 0.021$ \\
\hline C16:1/C16:0 ( $\triangle 9 \mathrm{D}-16)$ & & $0.02 \pm 0.02$ & $0.02 \pm 0.02$ & $0.02 \pm 0.01$ \\
\hline C18:1/C18:0 ( $\triangle 9 \mathrm{D}-18)$ & & $1.18 \pm 0.87$ & $1.17 \pm 0.66$ & $1.17 \pm 0.71$ \\
\hline C24:1/C18:1 & & $0.18 \pm 0.11^{*}$ & $0.12 \pm 0.08^{* * *}$ & $0.22 \pm 0.12$ \\
\hline C18:0/C16:0 & & $0.41 \pm 0.21^{* * *}$ & $0.48 \pm 0.21^{* *}$ & $0.57 \pm 0.17$ \\
\hline
\end{tabular}

Data obtained by GC are expressed as relative values (\%): mean \% of total FAs \pm SD; $\boldsymbol{\Sigma}$ SFA, sum of saturated fatty acids; $\boldsymbol{\Sigma}$ MUFA, sum of monounsaturated fatty acids; $\boldsymbol{\Sigma}$ PUFA, sum of polyunsaturated fatty acids; $\boldsymbol{\Sigma}$ UFA, sum of unsaturated fatty acids; C20:4/C20:3, $\Delta 5$ desaturation index; C18:3/C18:2, $\Delta 6$ desaturation index; C16:1/C16:0 and C18:1/C18:0, $\Delta 9$ desaturation index; $C 24: 1 / C 18: 1$ and $C 18: 0 / C 16: 0$, elongation index; w/o, without ${ }^{*} P<0.05 ;{ }^{* *} P<0.01$ and ${ }^{* *} P<0.001$ compared to controls. The Mann-Whitney test with Bonferroni correction was used 
IIEF-5 score and FAs profiles. Similarly, significantly different onset of age of VED was not found between the different FAs classes (data not shown).

\section{Discussion}

The physiopathology of diabetic ED is multifactorial and a number of associated risk factors are proposed [5], yet new molecular and cellular approaches are suspected to promote this complication. The present paper aims to investigate the effect of disturbed FAs metabolism and obvious risk factors on the susceptibility to VED in people with T2D.

Insulin has multiple physiological effects on the vascular tissues such as vasodilation, mainly due to its ability to increases eNOS expression and NO production via the activation of PI3K/Akt pathways [8]. However, IR prevents this increase [7]. The hypothesis that IR is a result of accumulation of intracellular lipid metabolites (e.g., FAs, diacylglycerol, fatty acyl CoAs) in endothelial cells, hepatocytes and skeletal muscle is supported by observations in patients and mouse models of lipodystrophy [22]. In fact, high SFAs amount affects the integrity, the fluidity and the structure of cell membranes leading to a decrease in the number of insulin receptors and an increase of IR severity [23] (Fig. 1).
In the present study, patients with VED displayed higher SFAs content associated with increased HOMAIR levels and lower concentration of NO than the other studied groups. Interestingly, this decline in NO levels was detected in T2D patients with VED (A) but not in those without VED (B), although both of the groups had IR. The severity of IR may play a crucial role in impaired NO bioactivity. As shown in our study, patients of group A developed diabetes during a longer period (Table 1) and had a greater amount in absolute SFAs concentration (Table 4) than patients of group B. This finding suggests that the length of the disease process, the excess of SFAs content and IR are the main culprits that affect the NO-cGMP pathway leading to changes in vascular tone and VSMCs function.

The synthesis of PUFAs requires several reactions of elongation catalyzed by the microsomal enzymes systems, essentially elongase, delta-5 $(\Delta 5 \mathrm{D})$ and delta-6 desaturases $(\Delta 6 \mathrm{D})[24]$. In fact, $\Delta 6 \mathrm{D}$ catalyzes the conversion of LA (C18:2 n-6) into GLA (18:3n-6), whereas elongase converts GLA (18:3 n-6) into DGLA (20:3 n-6). The outputs of DGLA and precisely its metabolite PGE1 activates soluble adenylyl cyclase (sAC), which increase cAMP concentration. Acting as a second messenger molecule, cAMP decrease intracellular calcium concentration and participate in VSMCs relaxation [10]. Our

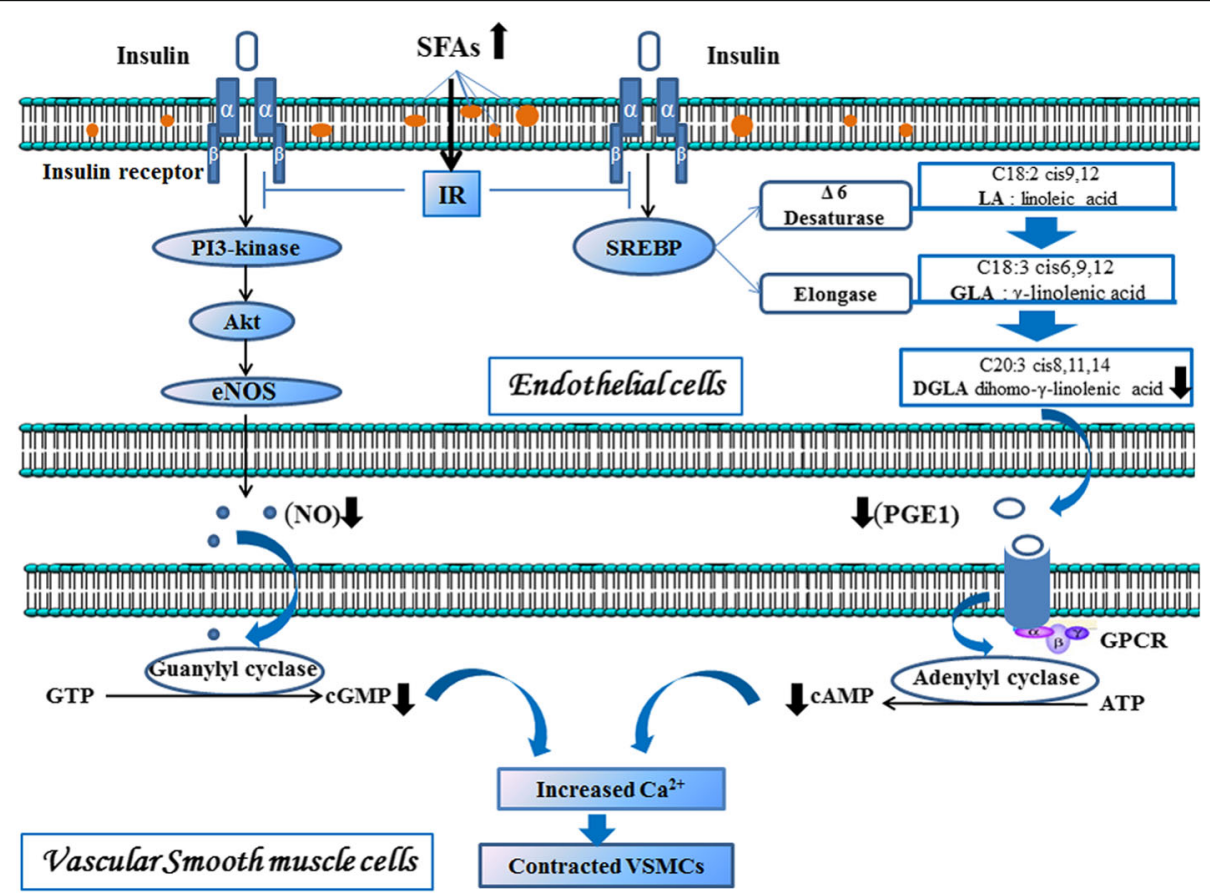

Fig. 1 Hypothetical interaction between disturbed FAs metabolism and IR leading to impaired penile erection. SFAs, Saturated Fatty Acids; IR, Insulin Resistance; PI3-kinase, Phosphatidylinositol 3-Kinase; SREBP, Sterol Regulatory Element Binding Protein; Akt, Protein kinase B; eNOS, Endothelial Nitric Oxide Synthase; PGE1, Prostaglandin E1; NO, Nitric Oxide; GPCR, G-Protein-Coupled Receptor; ATP, Adenosine Triphosphate; cAMP, cyclic Adenosine Monophosphate; GTP, Guanosine Triphosphate; cGMP, cyclic Guanosine Monophosphate; Ca ${ }^{2+}$, Calcium; VSMCs, Vascular Smooth Muscle 
study reveals that T2D patients with VED have significantly lower indexes of elongase and $\Delta 6$-desaturase activities than the control group as well as a significant decrease in DGLA levels. It thereby affirms that the stimulation of erection through the PGE1-cAMP pathway is down regulated. The suggested mechanism underlying the observed decrease in the index of elongase and $\triangle 6 \mathrm{D}$ activities is IR. In fact, insulin modulates the activities of some enzymes of the microsomal systems [25]. Insulin effects occur via the transcriptional activation of lipogenic transcription factors such as sterol regulatory element binding protein (SREBP), which increase the transcription of genes encoding enzymes of PUFAs synthesis such as $\Delta 6$-desaturase, acetyl-coenzyme A (CoA) carboxylase, fatty acid synthase, and the elongase [25, 26] (Fig. 1). Accordingly, IR plays an essential role in inhibiting the activity of these enzymes affecting afterwards DGLA synthesis and PGE1-mediated sAC activation.Diet constitutes a crucial aspect of the overall management of diabetes, which may prevent or ameliorate diabetes-related complications. In fact, recent studies suggested that PUFAs intake lowers the risk of vascular complications associated with diabetes such as retinopathy [27] and nephropathy [28]. A multicenter clinical trial revealed a significant improvement of 13 diabetic neuropathy parameters in T2D patients supplemented with gamma-linolenic acid for one year [29]. Moreover, it was demonstrated that an excess of SFAs content is positively correlated with IR and T2D risk, whereas long chain n-3 FAs such as EPA and DHA are protective $[18,30]$. These two PUFAs can be synthesized from ALA (18:3 n-3). Although RBCs levels of ALA are higher in patients of group A, a decrease in EPA and DHA concentrations is recorded. Research suggests that only a small amount of these two biologically important dietary FAs can be synthesized in the body from ALA process [31] and our study showed that the efficiency of the enzymatic reactions involved is rather low., Furthermore, Delarue and Guriec (2011) reported that diet rich in MUFAs, improved insulin sensitivity in patients with T2D [30]. MUFAs reduce plasma triglycerides, postprandial lipemia and markers of inflammation [30]. In the present study a significant reduction in erythrocytes levels of several $\dot{\omega} 9$ MUFAs were registered among T2D patients with VED. The findings herein reported obviously corroborate the fact that imbalanced diet fat intake may be involved in the physiopathology of VED in people with T2D. We believe that it would be interesting to test the potential beneficial effects of DGLA n-6 supplementation with adequate amounts of PUFAs (such as EPA and DHA) on modulating the risk of this disorder.

Nuclear transcription factors involved in the induction of cytokine genes are regulated by lipid mediators and products of lipid peroxidation [32]. MDA is a main $\omega 6$ PUFAs lipid peroxidation product that up-regulates IL-6 gene expression [32]. The stimulation of TNF- $\alpha / \mathrm{IL}-6$ signaling is a key mechanism underlying increased Endothelin-1 (ET-1) production and subsequent vasoconstriction effects [33]. ET-1 is one of the most powerful endogenous vasoconstrictors. Moreover, the levels of eNOS mRNA in human endothelial cells (ECs) are directly decreased by high concentrations of TNF- $\alpha$ [34]. There is evidence that cytokines activation in VSMCs hinders the production and/or activity of vasodilator mediators such as NO, bradykinin, prostacyclin and triggers vasoconstrictive mediators expression such as ET-1, thromboxane and angiotensin II [13]. In the present investigation, MDA and IL-6/TNF- $\alpha$ plasmatic levels are significantly higher and positively correlated in T2D patients with VED. Altogether, our results support a major role of inflammation in the pathogenesis of diabetic VED.

\section{Strengths and limitations}

FAs can be measured in various tissues and blood fractions such as RBCs, serum or plasma. FAs composition in membranes of RBCs may be superior to plasma or serum FAs for reflecting long-term FAs intake because of a slower turnover rate and less sensitivity to recent intake [1]. Hence, RBCs represent the most suitable model for comparing FAs classes with regard to their effect on metabolic disorders.

The limitations of the present study should be regarded. The sample size is adequate for statistical analysis but not large enough for epidemiological considerations. The measurement of desaturases activities in the leukocytes and SHBG (sex hormone binding globulin) level should be considered in future investigations. A dietary survey is recommended to support our findings.

\section{Conclusions}

The conducted study shows that balanced FAs metabolism is required for the regulation of key pathways involved in stimulating erection. Understanding the cellular mechanism(s) generating FAs dysregulation offers the prospect of better targeted and more effective therapeutic interventions for treatment and prevention of VED in men with T2D.

\footnotetext{
Acknowledgements

We would like to express our recognition to all participants in this study. We would like also to thank Dr. Imed Chraeif for his help in conducting GC analysis.

Funding

This work has been financially supported by the "Tunisian Ministry of Higher Education, Scientific Research and Technology".

Availability of data and materials

The dataset used and/or generated during the current study are available from the corresponding author on reasonable request.
} 


\section{Authors' contributions}

MBK conceived the idea of the manuscript, performed the experiments, analyzed the data, prepared tables, and wrote the paper. MBK, HB, SHA, MA participated in patients and controls' recruitment, MA, KJ contributed to reagents/materials/analysis tools and reviewed drafts of the paper, $\mathrm{MH}$ contributed to initial study concept, study design, and final editing of the paper and supervised the overall study. All authors read and approved the final manuscript.

\section{Ethics approval and consent to participate}

All participants gave consent to this study that was approved by the local Medical Ethics Committee of the Hedi Chaker Hospital of Sfax.

\section{Consent for publication}

Not applicable.

\section{Competing interests}

The authors declare that they have no competing interests.

\section{Publisher's Note}

Springer Nature remains neutral with regard to jurisdictional claims in published maps and institutional affiliations.

\section{Author details \\ 'Laboratory of Nutrition, Functional Food and Vascular Health, Department of Biochemistry, Faculty of Medicine, University of Monastir, Avenue Avicenne, 5019 Monastir, Tunisia. ${ }^{2}$ Unit of Obesity and Metabolic Syndrome, Department of Endocrinology, University Hospital Hedi Chaker of Sfax, Sfax, Tunisia. ${ }^{3}$ Biochemistry Laboratory, University Hospital Hedi Chaker of Sfax, Sfax, Tunisia.}

\section{Received: 28 August 2017 Accepted: 5 December 2017}

\section{Published online: 12 December 2017}

\section{References}

1. Sun Q, Ma J, Campos H, Hankinson SE, Hu FB. Comparison between plasma and erythrocyte fatty acid content as biomarkers of fatty acid intake in US women. Am J Clin Nutr. 2007;86:74-81.

2. Mann $\mathrm{Jl}$. Nutrition recommendations for the treatment and prevention of type 2 diabetes and the metabolic syndrome: an evidenced-based review. Nutr Rev. 2006:64:422-7.

3. Risérus $\mathrm{U}$, Willett WC, Hu FB. Dietary fats and prevention of type 2 diabetes. Prog Lipid Res. 2009;48:44-51.

4. Lebret T, Cour F. Impuissance: dysfonction érectile. EMC-Endocrinologie. 2005; 2:77-88.

5. Phé V, Rouprêt M. Erectile dysfunction and diabetes: a review of the current evidence-based medicine and a synthesis of the main available therapies. Diabetes Metab. 2012:38:1-13.

6. Sears B, Perry M. The role of fatty acids in insulin resistance. Lipids Health Dis. 2015:14:121-9.

7. Trussell JC, Legro RS. Erectile dysfunction: does insulin resistance play a part? Fertil Steril. 2007:88:771-8.

8. Muniyappa R, Sowers JR. Role of insulin resistance in endothelial dysfunction. Rev Endocr Metab Disord. 2013;14:5-12.

9. Ruiz Rubio JL, Hernández M, Rivera de los Arcos L, Martínez AC, GarcíaSacristán A, Prieto D. Mechanisms of prostaglandin E1-induced relaxation in penile resistance arteries. J Urol. 2004;171:968-73.

10. Onyango AN. The contribution of singlet oxygen to insulin resistance. Oxid Med Cell Longev. 2017:2017:8765972.

11. Das UN. Is erectile dysfunction a low-grade systemic inflammatory condition? Eur Heart J. 2007:28:642-3.

12. Yadav UC, Ramana KV. Regulation of NF-KB-induced inflammatory signaling by lipid peroxidation-derived aldehydes. Oxidative Med Cell Longev. 2013:4:1-11.

13. Sprague AH, Khalil RA. Inflammatory cytokines in vascular dysfunction and vascular disease. Biochem Pharmacol. 2009:78:539-52.

14. Das UN. Lipoxins, resolvins, and protectins in the prevention and treatment of diabetic macular edema and retinopathy. Nutrition. 2013:29:1-7.

15. Miranda EP, Mulhall JP. International index of erectile function erectile function domain vs the sexually health inventory for men: methodological challenges in the radical prostatectomy population. BJU Int. 2015;115:355-6.
16. Safarinejad MR, Khoshdel A, Shekarchi B, Taghva A, Safarinejad S. Association of the T-786C, G894T and 4a/4b polymorphisms of the endothelial nitric oxide synthase gene with vasculogenic erectile dysfunction in Iranian subjects. BJU Int. 2011:107:1994-2001.

17. Safarinejad MR, Safarinejad S, Shafiei N, Safarinejad S. G-protein b3 subunit gene $825 \mathrm{C} / \mathrm{T}$ polymorphism and its association with the presence, severity, and duration of vasculogenic erectile dysfunction. Fertil Steril. 2013;99:69-75.

18. Yoshioka T, Kwada K, Shimada T, Mori M. Lipid peroxidation in maternal and cord blood and protective mechanisms against elevated oxygen toxicity in the blood. Am J Obstet Gynecol. 1979;135:972-6.

19. Esterbauer H, Retteneder M, Striegl G, Waeg G, Ashy A, Sattler W, Jürgens G. Vitamin $\mathrm{E}$ and other lipophilic antioxidants protect LDL against oxidation. Eur J Lipid Sci Tech. 1989;91:316-24.

20. Folch J, Lees M, Sloane Stanley GH. A simple method for the isolation and purification of total lipids from animal tissues. J Biol Chem. 1957;226:497-509.

21. Zarrouk A, Riedinger JM, Ahmed SH, Hammami S, Chaabane W, Debbabi M, Ben Ammou S, Rouaud O, Frih M, Lizard G, Hammami M. Fatty acid profiles in demented patients: identification of hexacosanoic acid (C26:0) as a blood lipid biomarker of dementia. J Alzheimers Dis. 2015;44:1349-59.

22. Petersen KF, Shulman Gl. Etiology of insulin resistance. Am J Med. 2006;1 19:10-6.

23. Weijers RBM. Lipid composition of cell membranes and its relevance in type 2 diabetes mellitus. Curr Diabetes Rev. 2012:8:390-400.

24. Nakamura MT, Nara TY. Structure, function, and dietary regulation of delta6, delta5, and delta9 desaturases. Annu Rev Nutr. 2004:24:345-76.

25. Vaubourdolle M. Biochimie et Hématologie. 2nd ed. France: Wolters Kluwer; 2007. p. 220-1.

26. Flowers MT, Ntambi JM. Stearoyl-CoA desaturase and its relation to highcarbohydrate diets and obesity. Biochim Biophys Acta. 2009;1791:85-91.

27. Emily Y, Chew MD. Dietary intake of Omega-3 fatty acids from fish and risk of diabetic retinopathy. JAMA. 2017;317:2226-7.

28. Han E, Yun Y, Kim G, Lee Y, Wang HJ, Lee BW, Cha BS, Kim BS, Kang ES. Effects of Omega-3 fatty acid supplementation on diabetic nephropathy progression in patients with diabetes and Hypertriglyceridemia. PLoS One. 2016;11(5):e0154683

29. Keen H, Payan J, Allawi J, Walker J, Jamal GA, Weir Al, Henderson LM Bissessar EA, Watkins PJ, Sampson M, Gale EAM, Scarpello J, Boddie HG, Hardy KJ, Thomas PK, Misra P, Halonen JP. Treatment of diabetic neuropathy with -linolenic acid. Diabetes Care. 1993;16:8-15.

30. Delarue J, Guriec N. Fatty acids, insulin resistance, metabolic syndrome and type 2 diabetes. Médecine des maladies Métaboliques. 2011;5:253-6.

31. Hussein N, Ah-Sing E, Wilkinson P, Leach C, Griffin BA, Millward DJ. Longchain conversion of [13C]linoleic acid and alpha-linolenic acid in response to marked changes in their dietary intake in men. J Lipid Res. 2005;46:269-80.

32. Raghavan S, Subramaniyam G, Shanmugam N. Proinflammatory effects of malondialdehyde in lymphocytes. J Leukoc Biol. 2012;92:1055-67.

33. Zhou CC, Irani RA, Dai Y, Blackwell SC, Hicks MJ, Ramin SM, Kellems RE, Xia Y. Autoantibody-mediated IL-6-dependent endothelin-1 elevation underlies pathogenesis in a mouse model of preedlampsia. Immunol. 2011:186:6024-34.

34. Yan G, You B, Chen SP, Liao JK, Sun J. Tumor necrosis factor-alpha downregulates endothelial nitric oxide synthase mRNA stability via translation elongation factor 1-alpha 1. Circ Res. 2008:103:591-7.

\section{Submit your next manuscript to BioMed Central and we will help you at every step:}

- We accept pre-submission inquiries

- Our selector tool helps you to find the most relevant journal

- We provide round the clock customer support

- Convenient online submission

- Thorough peer review

- Inclusion in PubMed and all major indexing services

- Maximum visibility for your research

Submit your manuscript at www.biomedcentral.com/submit
) Biomed Central 